\title{
Correlation function and generalized master equation of arbitrary age
}

\author{
Paolo Allegrini, ${ }_{1}^{1}$ Gerardo Aquino, ${ }^{2, *}$ Paolo Grigolini, ${ }^{2,3,4}$ Luigi Palatella, ${ }^{5}$ Angelo Rosa, ${ }^{6}$ and Bruce J. West ${ }^{7}$ \\ ${ }^{1}$ INFM, unità di Como, Via Valleggio 11, 22100 Como, Italy \\ ${ }^{2}$ Center for Nonlinear Science, University of North Texas, P.O. Box 311427, Denton, Texas 76203-1427, USA \\ ${ }^{3}$ Dipartimento di Fisica dell'Università di Pisa and INFM, Via Buonarroti 2, 56127 Pisa, Italy \\ ${ }^{4}$ Istituto dei Processi Chimico Fisici del CNR Area della Ricerca di Pisa, Via G. Moruzzi 1, 56124 Pisa, Italy \\ ${ }^{5}$ Dipartimento di Fisica and Istituto dei Sistemi Complessi del CNR, Università di Roma "La Sapienza,” P.le A. Moro 2, \\ 00185 Rome, Italy \\ ${ }^{6}$ Institut de Mathématiques B, Faculté des Sciences de Base, École Polytechique Fédérale de Lausanne, 1015 Lausanne, Switzerland \\ ${ }^{7}$ Mathematics Division, Army Research Office, Research Triangle Park, North Carolina 27709, USA
}

(Received 17 September 2004; revised manuscript received 21 December 2004; published 10 June 2005)

\begin{abstract}
We study a two-state statistical process with a non-Poisson distribution of sojourn times. In accordance with earlier work, we find that this process is characterized by aging and we study three different ways to define the correlation function of arbitrary age of the corresponding dichotomous fluctuation. These three methods yield exact expressions, thus coinciding with the recent result by Godrèche and Luck [J. Stat. Phys. 104, 489 (2001)]. Actually, non-Poisson statistics yields infinite memory at the probability level, thereby breaking any form of Markovian approximation, including the one adopted herein, to find an approximated analytical formula. For this reason, we check the accuracy of this approximated formula by comparing it with the numerical treatment of the second of the three exact expressions. We find that, although not exact, a simple analytical expression for the correlation function of arbitrary age is very accurate. We establish a connection between the correlation function and a generalized master equation of the same age. Thus this formalism, related to models used in glassy materials, allows us to illustrate an approach to the statistical treatment of blinking quantum dots, bypassing the limitations of the conventional Liouville treatment.
\end{abstract}

DOI: 10.1103/PhysRevE.71.066109

PACS number(s): 02.50.Ey, 05.40.Fb, 05.20.-y, 05.60.Cd

\section{INTRODUCTION}

The phenomenon of aging has been known for a long time to be a property of spin glasses and polymers [1]. Part of the reason for the recent interest in the aging phenomenon has to do with the predicted breakdown of certain fundamental assumptions made in equilibrium statistical mechanics when applied to strongly disordered systems. For example, the Onsager principle [2], which is the relaxation of a perturbed system back to its equilibrium state described by an unperturbed autocorrelation function, is violated in anomalous diffusion leading to an anomalous relaxation. More recent papers on this phenomenon are devoted to studying aging in diffusion processes occuring in $d$-dimensional lattices [3], in low dimensional environments [4] and in the quantum dynamics of dissipative free particles [5]. Most recently [6,7] there has been some interest in the manifestation of aging in processes described by means of the continuous time random walk (CTRW) formalism [8].

The recent work of Ref. [9] establishes that the intermittent radiation-induced fluorescence of colloidal semiconductor quantum dots also yields aging. These new materials, under the influence of a radiation field generate intermittent fluorescence suggesting that the system jumps back and forth

\footnotetext{
*Corresponding author. Present address: Institute for Theoretical Physics, University of Amsterdam, Valckeniarstraat 65, 1018XE Amsterdam, the Netherlands. Email address: gaquino@science.uva.nl
}

from a "light on" to a "light off" state [10], with a nonPoisson distribution of times of sojourn in these two states. This is a quantum mechanical process that, in principle, should be described using a two-state master equation. However, the important work of Jung et al. [10] shows that the adoption of the ordinary Liouville approach to the density time evolution encounters serious difficulties, caused by the presence of aging. This difficulty is confirmed by the more recent work of Aquino et al. [11], who have evaluated the emission and absorption spectrum of this intermittent system using the CTRW formalism. For this reason, it is important to address the problem of the foundation of the master equation from the new perspective of trajectories (CTRW) rather than from that of densities, as in the fundamental work of Zwanzig [12]. The conflict between these two pictures, noticed for the first time by Bologna et al. [13], is far from being fully understood, and it is not yet clear if the controversy is due to the inadequacy of the known forms of nonMarkov master equations, derived from the Liouville-density method, or to an even more fundamental reason, namely, the breakdown of the generally accepted equivalence between the density and trajectory pictures. To shed light into this important issue, herein we plan to reexamine the issue of aging, widely discussed in the field of glassy materials, and the important mathematical treatment of this property made by Godrèche and Luck [14] as well, from the perspective of the generalized master equation (GME).

A natural way of formally expressing aging is through the correlation function of the stochastic variable of interest, $\xi$, which is expressed as an ensemble average indicated by the brackets 


$$
\frac{\left\langle\xi\left(t_{1}\right) \xi\left(t_{2}\right)\right\rangle_{t_{a}}}{\left\langle\xi^{2}\right\rangle} \equiv \Phi_{\xi}^{\left(t_{a}\right)}\left(t_{1}-t_{2}\right)
$$

As we shall see throughout this paper, the parameter $t_{a}$ denotes the time distance between the preparation and the observation process. The averaging brackets carry a subscript stressing that this is a $t_{a}$-old property, rather than the traditional aged property, only depending on $\left|t_{1}-t_{2}\right|$. The time difference indicates the stationarity of the underlying process, but the $t_{a}$ superscript denotes a dependence on the age of the process as well. In the case of a dichotomous variable obeying renewal theory, the exact expression for the Laplace transform of this age-dependent correlation function was found by Godrèche and Luck [14]. Their exact result was recently recovered by Margolin and Barkai [15] as a special case of a more general expression, since the latter authors do not require the condition that the two states of the dichotomous variable $\xi$ have the same waiting-time distribution. However, herein we make the same assumption as did Godrèche and Luck [14], and derive their result along the lines of the recent work of Ref. [7]. Allegrini et al. [7] noticed that in the non-Poisson case the well known GME of Kenkre, Montroll, and Shlesinger [16] becomes incompatible with the Onsager principle [2] and found a way to make the GME compatible with the aged condition. However, they [7] left unsolved the problem of deriving a GME of arbitrary age, which would be equivalent to making the Onsager principle compatible with an incompletely aged system. Herein we solve this problem and establish that this solution leads to the exact expression for the correlation function of arbitrary age found by Godrèche and Luck [14].

The GME derived herein does not have the same origin as those widely discussed in the literature [12]. The Zwanzig GME, in fact, one of the most popular master equations, is derived from a first principles procedure, starting from the statistical Liouville equation of the whole universe. The Zwanzig GME is a projection of this universal Liouville equation onto the Hilbert space of the system of interest. The approach used to derive the GME herein is based on the experimental observation of non-Poisson dichotomous signals. Examples of such signals include those produced by ionic channels [17] and by blinking quantum dots [10]. We build up a GME that is compatible with the experimentally determined non-Poisson nature of these processes, assuming the applicability of renewal theory. We leave open the question as to the source of randomness, but note in passing that the fluctuations in the system variable are generated by the environment. A simplified dynamical model has reproduced the essential statistical properties of the system of interest, in the case of infinitely aged GME [18]. In the case of arbitrary age, not only a first principle derivation is missing, a condition shared by the infinitely aged case: there is, to date, not even a heuristic derivation of the corresponding GME. Herein we provide a heuristic derivation of a GME with arbitrary age, one based on an exact expression for the corresponding distribution of sojourn times, and we use this result to derive an exact expression for the correlation function.
In concluding the Introduction, we point out that we rework the problem from a number of perspectives. We do this because each approach provides separate and distinct insights into the phenomenon described by a GME of arbitrary age. The first perspective adopted in this paper focuses on the derivation of a GME, based on the experimental observation of the time evolution of a trajectory, characterized by rare jumps from one state to another. The second perspective uses a Liouville-like approach to the time evolution of the variable of interest and its environment. No attempt is made to derive the former dynamic picture (trajectory) from the latter (probability density). The probability density perspective is characterized by infinite memory, yet, the statistical process under study is generated by random critical events, whose occurrence erases the memory of earlier events. Therefore, we think it is prudent to examine, yet again, the same process from a third perspective, one based on the direct observation of the sequence of rare random events.

We find that the use of three different perspectives is fruitful in shedding light on the recent observation made by Sokolov, Blumen, and Klafter [19]. Their results, in our opinion, imply the breakdown of certain well-established notions of equilibrium statistical mechanics, such as linear response theory as a prescription for predicting the effect of externally perturbing a system out of equilibrium.

The outline of the paper is as follows. In Sec. II we employ the reduced density perspective, namely, we build up the GME of arbitrary age. In Sec. III we study the time evolution of the total distribution density. In Sec. IV we use a third method, based on the probability of occurrence of the rare random events. We devote Sec. V to illustrate the physical connection between spin-glass and blinking quantum dot aging. Finally, in Sec. VI we make some concluding remarks.

\section{THE REDUCED DENSITY PERSPECTIVE}

This section is devoted to the application of the reduced density perspective to the construction of a GME. In the statistical physics literature this perspective implies a contraction on the total density matrix onto a prescribed subspace. Consequently, constructing the equation of motion of the total density matrix should be the first step. The reason why we reverse the perspective, thereby confining the total density treatment to the next section, is due to the fact that non-Poisson statistics has the effect of making the ordinary approach extremely difficult, if not impossible, as discussed in the companion papers $[18,20]$. The derivation of the GME is made possible by expressing the higher-order correlation functions in terms of the second-order correlation function, via simple expressions that are violated by the non-Poisson statistics. This is the reason why we derive the GME from the continuous time random walk (CTRW) of Montroll and Weiss $[8,21]$, rather than from the equation of motion for the total distribution density.

\section{A. From the generalized master equation to the age-dependent correlation function}

The reduced density matrix perspective is based on the adoption of the GME formally defined by 


$$
\frac{d}{d t} \mathbf{p}(t)=-\int_{0}^{t} \Phi\left(t-t^{\prime}\right) \mathbf{K} \mathbf{p}\left(t^{\prime}\right) d t^{\prime}
$$

where $\mathbf{p}(t)$ is the $m$-dimensional population vector of $m$ sites, so that its $i$ th component, $p_{i}(t)$, represents the probability of finding the walker at time $t$ in the $i$ th site. states. $\mathbf{K}$ is a transition matrix between the sites and $\Phi(t)$ is the memory kernel. We study the case where the fluctuating variable $\xi$ leading to this GME is dichotomous. This means that the GME has only two states and the matrix $\mathbf{K}$ reads

$$
\mathbf{K}=\left(\begin{array}{rr}
1 & -1 \\
-1 & 1
\end{array}\right) \text {. }
$$

The waiting-time distribution in either of the two states is denoted by $\psi(t)$. We establish the connection of the GME with the CTRW $[7,8]$ by relating the Laplace transform of the memory kernel $\Phi(t)$, given by $\hat{\Phi}(u)$, to the Laplace transform of the waiting-time distribution $\psi(t)$, given by $\hat{\psi}(u)$, as follows:

$$
\hat{\Phi}(u)=\frac{u \hat{\psi}(u)}{1-\hat{\psi}(u)} .
$$

We consider two distinct processes, that of preparation and that of observation. The preparation process establishes the initial conditions of the set of trajectories under study. A trajectory is a sequence of symbols + or - , specifying whether the system is in the state $|+\rangle$ or $|-\rangle$. We call the time interval with the system either entirely in the state $|+\rangle$ or entirely in the state $|-\rangle$ the laminar region. With Zumofen and Klafter [22], we assume that the preparation process, beginning at time $t=-t_{a}$, insures that all these trajectories begin with the system at the onset of a laminar region, either + or - .

The observation process begins at time $t=0 \geqslant-t_{a}$. The distribution of first sojourn times is denoted by $\psi_{t_{a}}(t)$ and is, in general, different from $\psi(t)$. In fact, the laminar region corresponding to the first sojourn time might have begun earlier than $t=0$. The exact expression for this time distribution is

$$
\psi_{t_{a}}(t)=\psi\left(t+t_{a}\right)+\sum_{n=1}^{\infty} \int_{0}^{t_{a}} d y \psi(y+t) \psi_{n}\left(t_{a}-y\right),
$$

where $\psi_{n}(\tau)$ denotes the probability that $n$ jumps occur during the time interval of length $\tau$, the last of which occurs at time $t=\tau$. As is well known, for a renewal process the waiting times for successively more jumps is given by the convolution

$$
\psi_{n}(t)=\int_{0}^{t} \psi_{n-1}\left(t^{\prime}\right) \psi_{1}\left(t-t^{\prime}\right) d t^{\prime},
$$

with $\psi(t) \equiv \psi_{1}(t)$. The $t_{a}$-old distribution of first sojourn time was discussed earlier in some detail [23]. However, a careful analysis of Eq. (5) can help the reader to realize the rationale behind this distribution of sojourn times. The first term on the right-hand side of Eq. (5) corresponds to the case when the first laminar region is extended in time more than the time interval $t_{a}$ between preparation and observation. The second term takes into account the cases when the last laminar region began after the preparation time, after a sequence of an arbitrarily large number of earlier laminar regions, the first of which, of course, begins at $t=-t_{a}$.

In the Poisson case, because of its unique functional form, there is no dependence of $\psi_{t_{a}}$ on $t_{a}$, and consequently no aging. In the non-Poisson case, on the contrary, the two waiting-time distributions, $\psi_{t}$ and $\psi(t)$, are identical only if $t_{a}=0$. In this case both CTRW and GME correspond to switching-on the observation process at the same time as the preparation process, and the connection between the two pictures is given by Eq. (4).

Allegrini et al. [7] proved that the GME is compatible with an infinitely aged CTRW, provided that the memory kernel $\Phi(t)$ is made compatible with an infinitely aged condition, characterized by a distribution of first sojourn times, which is infinitely aged. In this case $t_{a} \rightarrow \infty$ so that

$$
\hat{\Phi}_{\infty}(u)=\frac{u \hat{\psi}_{\infty}(u)}{1+\hat{\psi}(u)-2 \hat{\psi}_{\infty}(u)},
$$

where

$$
\psi_{\infty}(t)=\frac{1}{\langle\tau\rangle} \int_{t}^{\infty} d t^{\prime} \psi\left(t^{\prime}\right)
$$

It is straigthforward to extend the calculations of Ref. [7] to the case of an arbitrarily $t_{a}$-old system, so that Eq. (7) is replaced with

$$
\hat{\Phi}_{t_{a}}(u)=\frac{u \hat{\psi}_{t_{a}}(u)}{1+\hat{\psi}(u)-2 \hat{\psi}_{t_{a}}(u)},
$$

where $\hat{\psi}_{t_{a}}(u)$ is the Laplace transform of $\psi_{t_{a}}(t)$.

Using the GME with the $t_{a}$-old memory kernel, we define the age-dependent correlation function $\Phi_{\xi}^{\left(t_{a}\right)}(t)$ through its Laplace transform, as follows:

$$
\hat{\Phi}_{\xi}^{\left(t_{a}\right)}(u)=\frac{1}{u+2 \hat{\Phi}_{t_{a}}(u)} .
$$

This prescription corresponds to setting

$$
\Phi_{\dot{\xi}}^{\left(t_{a}\right)}(t)=\frac{p_{1}(t)-p_{2}(t)}{p_{1}(0)-p_{2}(0)},
$$

as one can easily check by Laplace transforming both sides and using the GME with the $t_{a}$-old memory kernel (see Sec. II B). old memory kernel. In other words, the correlation function of arbitrary age mirrors the extension of the Onsager principle, which is usually limited to infinitely aged systems [7], to physical conditions of arbitrary age. It has to be pointed out that at this stage there is no guarantee that the Onsager principle of arbitrary age holds true. However, in Sec. II B we show that Eq. (10) yields the exact result of Godrèche and Luck [14] for the corresponding correlation function. Furthermore, in Sec. II C we establish the same 
result using arguments based on trajectories rather than densities, thereby affording an independent construction of the exact expression for the $t_{a}$-old correlation function. All this can be thought of as a compelling demonstration of the correctness of Eq. (11), extending the Onsager principle to conditions of arbitrary age.

\section{B. Derivation of the exact expression proposed by Godrèche and Luck: The probability perspective}

To establish that the proposed approach yields the exact expression of Godrèche and Luck [14], let us express the $t_{a}$-old correlation function through the probability vector $\mathbf{p}$ $\equiv\left(p_{1}, p_{2}\right)$ for the dichotomous variable $\xi= \pm 1$ to have either positive (state 1 ) or negative (state 2 ) values. We have that

$$
\begin{aligned}
\Phi_{\xi}^{\left(t_{a}\right)}(t)= & \frac{\langle\xi(0) \xi(t)\rangle_{t_{a}}}{\left\langle\xi^{2}\right\rangle}=p_{1}(0) p_{1}(t \mid 1, t=0)+p_{2}(0) p_{2}(t \mid 2, t=0) \\
& -p_{1}(0) p_{2}(t \mid 1, t=0)-p_{2}(0) p_{1}(t \mid 2, t=0),
\end{aligned}
$$

where $p_{j}(t \mid k, t=0)$ is the conditional probability that the variable $\xi$ is in the state $j$ at time $t$, given that at time $t=0$ it was in the state $k$. This means that $p_{j}(t \mid k, t=0)$ is obtained letting those trajectories evolve that at time $t=0$ had $\xi$ in the state $k$. For a straightforward evaluation of $p_{j}(t \mid k, t=0)$, we use the GME formalism, adapted to the $t_{a}$-old system, and we take into account the initial condition $p_{i}(0 \mid k, t=0)=\delta_{i, k}$. According to the GME the components of the conditional probability vector are determined by

$$
\frac{d}{d t} p_{j}(t \mid k, t=0)=-\int_{0}^{t} d t^{\prime} \Phi_{t_{a}}\left(t-t^{\prime}\right) \sum_{i=1}^{2} K_{j i} p_{i}\left(t^{\prime} \mid k, t=0\right),
$$

with $\hat{\Phi}_{t_{a}}(u)$ given by Eq. (9) and the elements of $\mathbf{K}$ given by Eq. (3).

By Laplace transforming (13) and doing some algebra, we obtain

$$
\hat{p}_{j}(u \mid k, 0)=\sum_{i=1}^{2}\left[u \mathbf{I}+\hat{\Phi}_{t_{a}}(u) \mathbf{K}\right]_{j i}^{-1} p_{i}(0 \mid k, 0) .
$$

Defining the matrix

$$
\mathbf{J}=\left[u \mathbf{I}+\hat{\Phi}_{t_{a}}(u) \mathbf{K}\right]^{-1}=\left(\begin{array}{cc}
\frac{u+\hat{\Phi}_{t_{a}}(u)}{u\left[u+2 \hat{\Phi}_{t_{a}}(u)\right]} & \frac{\hat{\Phi}_{t_{a}}(u)}{u\left[u+2 \hat{\Phi}_{t_{a}}(u)\right]} \\
\frac{\hat{\Phi}_{t_{a}}(u)}{u\left[u+2 \hat{\Phi}_{t_{a}}(u)\right]} & \frac{u+\hat{\Phi}_{t_{a}}(u)}{u\left[u+2 \hat{\Phi}_{t_{a}}(u)\right]}
\end{array}\right),
$$

and using the initial condition $p_{i}(0 \mid k, t=0)=\delta_{i, k}$, we obtain for the Laplace transform of the conditional probability vector the following expression:

$$
\hat{p}_{j}(u \mid k, 0)=\frac{\left[u+\hat{\Phi}_{t_{a}}(u)\right] \delta_{j, k}+\hat{\Phi}_{t_{a}}(u)\left(\delta_{j, k+1}+\delta_{j, k-1}\right)}{u\left[u+2 \hat{\Phi}_{t_{a}}(u)\right]} .
$$

Using Eq. (15) we can Laplace transform (12) to obtain

$$
\begin{aligned}
\hat{\Phi}_{\xi}^{\left(t_{a}\right)}(u)= & p_{1}(0) \frac{u+\hat{\Phi}_{t_{a}}(u)}{u\left(u+2 \hat{\Phi}_{t_{a}}(u)\right)}+p_{2}(0) \frac{u+\hat{\Phi}_{t_{a}}(u)}{u\left(u+2 \hat{\Phi}_{t_{a}}(u)\right)} \\
& -p_{1}(0) \frac{\hat{\Phi}_{t_{a}}(u)}{u\left(u+2 \hat{\Phi}_{t_{a}}(u)\right)}-p_{2}(0) \frac{\hat{\Phi}_{t_{a}}(u)}{u\left(u+2 \hat{\Phi}_{t_{a}}(u)\right)} .
\end{aligned}
$$

We note that the probability is normalized, $p_{1}(0)+p_{2}(0)=1$. Thus, it follows that

$$
\begin{aligned}
\hat{\Phi}_{\xi}^{\left(t^{\prime}\right)}(u)= & {\left[\frac{u+\hat{\Phi}_{t_{a}}(u)}{u\left(u+2 \hat{\Phi}_{t_{a}}(u)\right)}-\frac{\hat{\Phi}_{t_{a}}(u)}{u\left(u+2 \hat{\Phi}_{t_{a}}(u)\right)}\right] } \\
& \times\left[p_{1}(0)+p_{2}(0)\right] \\
= & \frac{1}{u+2 \hat{\Phi}_{t_{a}}(u)},
\end{aligned}
$$

confirming the correctness of the definition introduced in Eq. (10). Substituting (9) into (17) we obtain

$$
\left.\hat{\Phi}_{\xi}^{\left(t_{a}\right)}(u)=\frac{1}{u\left[1+2 \frac{\hat{\psi}_{t_{a}}(u)}{1+\hat{\psi}(u)-2 \hat{\psi}_{t_{a}}(u)}\right]}\right]=\frac{1}{u}\left[1-\frac{2 \hat{\psi}_{t_{a}}(u)}{1+\hat{\psi}(u)}\right],
$$

which coincides with the results of Godrèche and Luck [14]. Furthermore, the ratio of the differences in probability is determined in Laplace space by

$$
\frac{\hat{p}_{1}(u)-\hat{p}_{2}(u)}{p_{1}(0)-p_{2}(0)}=\sum_{i=1}^{2} \frac{\left(J_{1 i}-J_{2 i}\right) p_{i}(0)}{p_{1}(0)-p_{2}(0)}=\frac{1}{u+2 \hat{\Phi}_{t_{a}}(u)}=\hat{\Phi}_{\xi}^{\left(t_{a}\right)}(u) \text {. }
$$

As pointed out earlier, Eq. (19) means that one can extend the Onsager principle from the infinitely aged systems, for which Onsager originally defined it, to systems of any age. In the latter case the relaxation is proportional to the $t_{a}$-old correlation function, not to the infinitely old, or equilibrium, correlation function. In summary, we discovered an Onsager principle of arbitrary age, at least in the special case of the dichotomous variables considered in this paper.

\section{Derivation of the exact expression proposed by Godrèche and Luck: The trajectory perspective}

It is possible to again derive the exact result of Eq. (18) from a different perspective, which will allow us, in Sec. IV, to propose an analytic expression for the $t_{a}$-old correlation 
function as a function of time. This expression, as we shall see, is not exact, but it is shown numerically to be a very good approximation to the exact result.

The usual method of connecting the correlation function $\Phi_{\xi}$ to the waiting-time distribution, within a trajectory perspective, is to introduce a theoretical waiting-time distribution, $\psi^{*}(t)$, which cannot be observed directly. In fact, the experimental waiting-time distribution, namely, the distribution of times with alternate signs, denoted by us as $\psi(t)$, is obtained from the theoretical waiting-time distribution, $\psi^{*}(t)$, by adopting the following procedure. We divide the time axis into bins, whose size is determined by the waiting-time distribution $\psi^{*}(t)$. Then, these bins are assigned either the value 1 or the value -1 , by tossing a coin to make the decision. It is evident that the intervals along the time axis with the same sign, are larger than the time bins determined by $\psi^{*}(t)$, since two or more consecutive coin tossings might have produced the same sign. It is shown [22] that the Laplace transform of $\psi$ is connected to the Laplace transform of $\psi^{*}$ via the relation

$$
\hat{\psi}(u)=\frac{\hat{\psi}^{*}(u)}{2-\hat{\psi}^{*}(u)} .
$$

Let us use the term event to denote the coin tossing introduced above. The expression (20) is the result of summing over all possibilities of not changing sign with a coin toss, which turns out to be a geometrical series in the Laplace representation. The correlation function $\Phi_{\xi}$ and the theoretical waiting-time distribution function $\psi^{*}$ are connected through the relation

$$
\Phi_{\xi}(t)=\frac{\int_{t}^{\infty}(t-\tau) \psi^{*}(\tau) d \tau}{\langle\tau\rangle},
$$

where the average waiting time is given by

$$
\langle\tau\rangle \equiv \int_{0}^{\infty} \tau \psi^{*}(\tau) d \tau
$$

Equation (21) determines that the correlation function $\Phi_{\xi}(t)$ is equal to the probability of finding a window of length $t$ without internal events.

The same result can be immediately recovered using

$$
\Phi_{\xi}(t)=\Psi_{\infty}^{*}(t) \equiv \int_{t}^{\infty} d t^{\prime} \psi_{\infty}^{*}\left(t^{\prime}\right)=\frac{1}{\langle\tau\rangle} \int_{t}^{\infty} d t^{\prime} \int_{t^{\prime}}^{\infty} d t^{\prime \prime} \psi^{*}\left(t^{\prime \prime}\right) .
$$

We note that, see Ref. [7],

$$
\psi_{\infty}^{*}(t) \equiv \frac{1}{\langle\tau\rangle} \int_{t}^{\infty} d t^{\prime} \psi^{*}\left(t^{\prime}\right)
$$

is the infinitely aged waiting-time distribution. Actually, Eq. (23) is the infinitely aged correlation function, a special case of the more general prescription

$$
\Phi_{\xi^{\prime}}^{\left(t_{a}\right)}(t)=\Psi_{t_{a}}^{*}(t) \equiv \int_{t}^{\infty} d t^{\prime} \psi_{t_{a}}^{*}\left(t^{\prime}\right)=1-\int_{0}^{t} d t^{\prime} \psi_{t_{a}}^{*}\left(t^{\prime}\right)
$$

It is straightforward to show that the $t_{a}$-old experimental waiting-time distribution and the $t_{a}$-old theoretical waitingtime distribution are connected through the following sum of convolutions:

$$
\psi_{t_{a}}(t)=\frac{1}{2} \psi_{t_{a}}^{*}(t) \star\left\{\delta(t)+\frac{1}{2} \psi^{*}(t)+\frac{1}{2} \psi^{*}(t) \star \frac{1}{2} \psi^{*}(t)+\cdots\right\},
$$

where the symbol $\star$ denotes time convolution. In fact, after a first interval of time followed by a coin toss with no change of sign, determined by $\psi_{t_{a}}^{*} / 2$, the next intervals of time with no change of sign according to the coin tossing prescription, are determined by $\psi^{*} / 2$. The sum of the convolutions takes into account all the possible sequences of intervals of time with no change of sign before a change of sign of the variable $\xi$ eventually occurs, and gives as final result the distribution for a first observed sojourn time $t$ of the variable $\xi$ in one of its two states, that is $\psi_{t_{a}}(t)$.

Thus by summing the geometric series in the Laplace variables, from (26) we obtain

$$
\hat{\psi}_{t_{a}}(u)=\frac{\hat{\psi}_{t_{a}}^{*}(u)}{2-\hat{\psi}^{*}(u)} .
$$

Using Eq. (20) we write Eq. (27) as

$$
\hat{\psi}_{t_{a}}^{*}(u)=\frac{2 \hat{\psi}_{t}(u)}{1+\hat{\psi}(u)} .
$$

By Laplace transforming Eq. (25) and using Eq. (28), we obtain

$$
\hat{\Phi}_{\xi}^{\left(t_{a}\right)}(u)=\frac{1-\hat{\psi}_{t_{a}}^{*}(u)}{u}=\frac{1}{u}\left[1-\frac{2 \hat{\psi}_{t_{a}}(u)}{1+\hat{\psi}(u)}\right],
$$

namely, we again recover the exact result of Godrèche and Luck given by Eq. (18). This establishes the equivalence of the trajectory and GME prescriptions for this process.

\section{Generalized master equation of arbitrary age}

We are now in a position to make a preliminary balance of the results obtained so far. The first is that we have generalized the result of an earlier paper [7], in that we have derived the GME of arbitrary age

$$
\frac{d}{d t} \mathbf{p}(t)=-\int_{0}^{t} \Phi_{t_{a}}\left(t-t^{\prime}\right) \mathbf{K} \mathbf{p}\left(t^{\prime}\right) d t^{\prime},
$$

whose memory kernel $\Phi_{t_{a}}(t)$ is defined though its Laplace transform by means of Eq. (9). We have also shown that the Onsager principle, valid for infinitely aged systems, can be extended to conditions of any age, and that this extension 
allows us to derive an exact expression for the $t_{a}$-old correlation function. However, the analytic results obtained so far are in the Laplace domain. It is desirable to achieve them in the time domain, as well. We now turn our attention to the latter.

\section{THE LIOUVILLE-LIKE APPROACH}

In this section we derive another expression for the $t_{a}$-old correlation function adopting a perspective where aging is determined by the out of equilibrium bath for the variable of interest, $\xi$. There might exist conditions, as we shall see, where equilibrium is not even allowed. The expression for the $t_{a}$-old correlation function afforded by this perspective is exact, and is thus equivalent to the Godrèche and Luck expression of Eq. (18). However, the exact expression is implicit, rather than explicit, and is therefore more convenient for the numerical calculations done subsequently.

Here we adopt the perspective of earlier work $[13,20]$ to account for the aging effects characterizing the fluctuations of the dichotomous variable $\xi$. These fluctuations occur while the environment of the variable, $\xi$, slowly drifts. This drifting process is extended over time, and could lead to circumstances where it is not possible to attain equilibrium asymptotically. In keeping with the jargon of statistical mechanics the environmental or "irrelevant" variable is called $y$ and in the model moves in the interval $I=[0,2]$. In the semiinterval $[0,1]$, we use the equation of motion for the probability density

$$
\frac{\partial p(y, t)}{\partial t}=-\lambda \frac{\partial}{\partial y} y^{z} p(y, t) .
$$

This is the motion determined by a potential, with the minimum at $y=1$, in the over-damped case. In the interval $[1,2]$, the overdamped potential is the mirror image of the potential acting on the left interval. Consequently, if the initial condition is located in the internal part of the interval $[0,1]$, the particle moves, from the left to the right, with a deterministic motion, towards $y=1$. If the particle is initially located in the interior of the interval [1,2], it moves deterministically from the right to the left. When the particle reaches the potential minimum, it is injected back, with equal probability, into any of the points of the interval $I$ excluding $y=0, y=1$ and $y=2$. The time spent by $y$ within $I$ corresponds to sojourning in one of the two states of the variable $\xi$, either $|+\rangle$ or $|-\rangle$. The instant of back injection corresponds to the choice of the new state and, with equal probability, this is either the same state or the other state. The variable $y$ represents the environment of the variable $\xi$, and its initial distribution is given by the state of the bath. The corresponding waiting-time distribution between two consecutive back injections is

$$
\psi^{*}(t)=(\mu-1) \frac{T^{\mu-1}}{(t+T)^{\mu}},
$$

where the index $\mu$ is related to $z$ of Eq. (31) by

$$
\mu \equiv \frac{z}{z-1},
$$

and the parameter $T$, characterizing the waiting-time distribution of Eq. (32) is

$$
T \equiv \frac{\mu-1}{\lambda},
$$

in accordance with the normalization constraint. The authors of Refs. $[7,18]$ have shown that the essential properties of the dichotomous non-Poisson fluctuation can be accounted for by limiting ourselves to this simplified picture, involving only the semi-interval $[0,1]$. In this simple picture the aging process is described by

$$
\frac{\partial p(y, t)}{\partial t}=-\lambda \frac{\partial}{\partial y} y^{z} p(y, t)+\lambda p(1, t),
$$

which takes into account the back injection into the semiinterval, occurring with uniform probability, when the particle reaches the point $y=1$. For simplicity, but with no loss of generality, we fix $\lambda=1$.

Using the results of Ref. [20] the $t_{a}$-old correlation function is evaluated as follows. The bath is prepared at time $t$ $=-t_{a}$. This means that at time $t=-t_{a}$ the distribution of $y$ within the interval $I$ is flat. In the case $z<2$ this distribution tends to the equilibrium distribution, $p_{e q} \propto 1 / y^{z-1}$. If $z>2$ this distribution diverges, thereby implying that the distribution approaches the Dirac delta function located at $y=0$. This is the nonstationary condition, the condition where equilibrium is not allowed, and only a condition of eternal drift is admitted. Suppose this distribution evolve for a time $t_{a}$, without our observing it. This means that we begin the observation process when the system has a new distribution, different from the initial flat distribution, and determined by its time evolution from $t=-t_{a}$ to $t=0$, described by Eq. (35). For $t$ $\geqslant 0$ Eq. (35) is replaced with Eq. (31)

$$
\frac{\partial p(y, t)}{\partial t}=-\frac{\partial}{\partial y} y^{z} p(y, t),
$$

namely the back injection process is stopped, thereby implying that the population decreases.

The theory of Ref. [20] relates the probability solution to the Liouville-like equation to the $t_{a}$-old correlation function as follows:

$$
\Phi_{\xi}^{\left(t_{a}\right)}(t)=\int_{0}^{1} d y p(y, t) .
$$

Note that the initial condition $p(y, 0)$ is obtained from Eq. (35) moving from the flat distribution at $t=-t_{a}$. The time evolution, corresponding to the observation process, is determined by Eq. (36). This simple picture is a fair representation of the description made in terms of trajectories in Sec. II. The fact that the norm of $p(y, t)$, when $p(y, t)$ is described by Eq. (36), is not conserved, reflects the occurrence of jumps from one state to the other, making the population of a given state decrease. Let us remark that the correlation function is determined by the antisymmetric part of the whole 
distribution [20]. This formal condition corresponds to observing the time evolution of $p(y, t)$, with $y$ ranging from $y$ $=0$ to $y=1$, under the action of Eq. (31), with no back injection process. The process of back injection is essential to determine the correlation function of arbitrary age. However, once the parameter $t_{a}$ is fixed, and with it the age of the correlation function, the evaluation of the correlation function is done by imagining the bath frozen in the distribution corresponding to this fixed age. This is the reason we use Eq. (31) to determine the correlation function rather than Eq. (35).

The process of back injection is essential for the slow environmental drift, but the correlation time of age $t_{a}$ is determined by the age-fixed condition, the bath being $t_{a}$ old, and keeping this age forever.

Our goal is to calculate the correlation function of arbitrary age. According to Eq. (37), this goal requires that we evaluate $p(y, t)$, first. To carry out this calculation we follow the procedure illustrated in detail in Ref. [24]. The time evolution from $t=-t_{a}$ to $t<0$ is described by Eq. (35), yielding for the solution

$$
\begin{aligned}
p(y, t)= & \frac{1}{\left[1+(z-1)\left(t+t_{a}\right) y^{z-1}\right]^{z /(z-1)}} \\
& +\int_{-t_{a}}^{t} \frac{p(1, \tau) d \tau}{\left[1+(z-1)(t-\tau) y^{z-1}\right]^{z /(z-1)}},
\end{aligned}
$$

with $p(1, \tau)$ determined, in turn, by Eq. (35).

As already stated, for $t>0, p(y, t)$ is governed by Eq. (36), so that its solution is, given the initial value,

$$
p(y, t)=\left.\frac{p(\eta, 0)}{\left[1+(z-1) y^{z-1} t\right]^{z /(z-1)}}\right|_{\eta=y /\left[1+(z-1) y^{z-1} t\right]^{1 /(z-1)}} .
$$

Now, we can calculate the final expression for the agedependent correlation function given by Eq. (37). After some simple algebra we obtain the following expression:

$$
\begin{aligned}
\Phi_{\xi}^{\left(t_{a}\right)}(t)= & \frac{1}{\left[1+(z-1)\left(t+t_{a}\right)\right]^{1 /(z-1)}} \\
& +\int_{-t_{a}}^{0} \frac{p(1, \tau) d \tau}{[1+(z-1)(t-\tau)]^{1 /(z-1)}} \\
= & \Psi^{*}\left(t+t_{a}\right)+\int_{-t_{a}}^{0} \frac{p(1, \tau) d \tau}{[1+(z-1)(t-\tau)]^{1 /(z-1)}} \\
= & \Psi^{*}\left(t+t_{a}\right)+\left[1-\Psi^{*}\left(t_{a}\right)\right] \\
& +\int_{-t_{a}}^{0} \frac{p(1, \tau) d \tau}{[1+(z-1)(t-\tau)]^{1 /(z-1)}} \\
& -\int_{-t_{a}}^{0} \frac{p(1, \tau) d \tau}{[1-(z-1) \tau]^{1 /(z-1)}} .
\end{aligned}
$$

Note that in Eq. (40) we are using the definition

$$
\Psi^{*}(t)=\int_{t}^{\infty} \psi^{*}(\tau) d \tau
$$

This notation for the survival probability is also used in Sec. IV A. We notice that, $t>0$ and $p(1, t)$ is calculated from Eq. (35) with $y=1$ and $-t_{a}<t<0$. This is an exact expression that turns out to be convenient to numerically check the prescriptions of Sec. IV.

It is worth using the perspective of this section to shed further light into the problem under discussion in this paper. Let us notice the following.

If the age vanishes $t_{a} \rightarrow 0$, then the $t_{a}$-old correlation function reduces to the probability of no event occuring $\Phi_{\xi}^{\left(t_{a}\right)}(t)$ $\rightarrow \Psi^{*}(t)$.

If the age increases without limit $t_{a} \rightarrow \infty$, then the $t_{a}$-old correlation function reduces to a known result $\Phi_{\xi}^{\left(t_{a}\right)}(t)$ $\rightarrow \Phi_{\xi}(t)$, where

$$
\Phi_{\xi}(t)=\frac{1}{[1+(z-1) t]^{(2-z) /(z-1)}}
$$

is the usual correlation function calculated at equilibrium. So, we must observe a crossover between two distinct regimes. Moreover, we anticipate that we recover these results from a different perspective in Sec. IV.

The perspective adopted in this section makes it easier to understand, on physical grounds, why the non-Poisson condition produces aging. The Poisson condition corresponds to $z=1$ in the nonlinear dynamical process of Eq. (35), and in this case the distribution is always flat. Aging is the process of slow regression of the bath variable to equilibrium, a process that can, in principle, last forever. With this representation it is possible to study the correlation function of arbitrary age also in the case $z>2$. Allegrini et al. [20] give a detailed discussion of the effect of external perturbations.

In the case $z<2$ equilibrium is possible. An external perturbation can be used to create a condition whereby the system is out of equilbrium. The observation of the population difference between the two states, $\xi=1$ and $\xi=-1$, is equivalent to determining the correlation function. Note that the initial condition that we use to study the time evolution of $p(y, t)$ during the observation process, Eq. (36), is the antisymmetric distribution of the interval $[0,2]$ folded into $[0,1]$ (see Ref. [20]). It is evident that in practice the equilibrium correlation function is never observed. In fact, the external perturbation should create a nonsymmetric initial condition in the whole interval $[0,2]$. The antisymmetric portion of this distribution, folded into the interval $[0,1]$, should create the same out of equilibrium distribution as that produced by folding in the same way the anti-symmetric portion of the equilibrium distribution. As pointed out in Ref. [20], this is impossible to do in practice with a a perturbation lasting for a finite time, and, thus, with any realistic experimental procedure.

\section{AN ANALYTICAL EXPRESSION OF THE CORRELATION FUNCTION OF ARBITRARY AGE}

The exact expressions, Eq. (18) and Eq. (29), allow us to determine the dependence of the age-dependent correlation 
function on time via inverse Laplace transforms. This inversion is done numerically since we are unable to carry out direct analytic inversion of the Laplace expression. There is therefore the need to find an analytical expression for $\Phi_{\xi}^{\left(t_{a}\right)}(t)$, even if the price we pay is an approximation and the loss of exactness. This section is devoted to the derivation of an accurate, albeit approximate, analytical expression for the correlation function of arbitrary age.

\section{A. An exact implicit expression for the correlation function of arbitrary age}

We write $\Phi_{\xi}^{\left(t_{a}\right)}(t)$, namely the probability of not finding events inside the interval $(0, t)$, given the occurrence of an event at time $-t_{a}$, in the following implicit form:

$$
\Phi_{\xi}^{\left(t_{a}\right)}(t)=\Psi^{*}\left(t+t_{a}\right)+\int_{0}^{t_{a}} d t_{l} \psi^{*}\left(t_{a}-t_{l}\right) \Phi_{\xi}^{\left(t_{l}\right)}(t) .
$$

This is the probability of finding an event at a time larger than $t$ from the preceding one. The right-hand side of Eq. (43) is the sum of two terms, the first corresponds to no event occurring in the whole interval $\left(-t_{a}, t\right)$ and the second term takes into account the possibility of at least one event occurring between $-t_{a}$ and 0 . The instant $-t_{l}$ signals the first of these events (or the event, if there is only one). The cointossing procedure has the double effect of rejuvenating the process (the correlation function now has age $t_{l}$ ) and of factoring the two probability functions inside the integral.

It is interesting to notice that Eq. (43) is exact, which can be verified by differentiating both sides of the equation, and making use of (25) and (41). This procedure yields

$$
\psi_{t_{a}}^{*}(t)=\psi^{*}\left(t+t_{a}\right)+\int_{0}^{t_{a}} d t_{1} \psi^{*}\left(t_{a}-t_{1}\right) \psi_{t_{1}}^{*}(t)
$$

namely an implicit (but analytic) expression for $\psi_{t_{a}}^{*}$. A straightforward iteration of (44) leads to

$$
\begin{aligned}
\psi_{t_{a}}^{*}(t)= & \psi^{*}\left(t+t_{a}\right)+\int_{0}^{t_{a}} d t_{1} \psi^{*}\left(t_{a}-t_{1}\right) \psi^{*}\left(t+t_{1}\right) \\
& +\int_{0}^{t_{a}} d t_{1} \psi^{*}\left(t_{a}-t_{1}\right) \int_{0}^{t_{1}} d t_{2} \psi^{*}\left(t_{1}-t_{2}\right) \psi^{*}\left(t+t_{2}\right) \\
& +\int_{0}^{t_{a}} d t_{1} \psi^{*}\left(t_{a}-t_{1}\right) \int_{0}^{t_{1}} d t_{2} \psi^{*}\left(t_{1}-t_{2}\right) \\
& \times \int_{0}^{t_{2}} d t_{3} \psi^{*}\left(t_{2}-t_{3}\right) \psi^{*}\left(t+t_{3}\right)+\cdots
\end{aligned}
$$

i.e., a sum over all possibilities of finding events at both times $t$ and $-t_{a}$ with no events between 0 and $t$. This is exactly the definition of $\psi_{t_{a}}^{*}(t)$. In detail, the first term in the right-hand side of (45), corresponds to the case in which no event occurs in the interval $I \equiv\left(-t_{a}, 0\right]$, while all further terms refer, respectively, to one event at $t_{1}$ in $I$, to two events, at $t_{1}$ and $t_{2}$, in $I$, and so on, with $t_{1}<t_{2}<t_{3}<\cdots$. Note that $\psi_{t_{a}}^{*}(t)$ is obtained from $\psi^{*}(t)$ in the same way $\psi_{t_{a}}(t)$ is ob- tained from $\psi(t)$ : in fact, if in Eq. (5) we make the substitution $\psi(t) \rightarrow \psi^{*}(t)$, we obtain exactly Eq. (45), i.e., the definition of $\psi_{t_{a}}^{*}(t)$.

\section{B. Equivalence with the exact expression of Godrèche and Luck}

As we said, Eq. (43) is exact. Consequently, this equation is expected to be equivalent to the exact expression proposed by Godrèche and Luck in Ref. [7]. In this subsection, as a double check, we prove that Eq. (43) is in fact equivalent to the expression of Godrèche and Luck. and To do that, let us differentiate both sides of this equation with respect to $t$ to obtain

$$
\frac{d}{d t} \Phi_{\xi}^{\left(t_{a}\right)}(t)=-\psi^{*}\left(t+t_{a}\right)+\int_{0}^{t_{a}} d t_{l} \psi^{*}\left(t_{a}-t_{l}\right) \frac{d}{d t} \Phi_{\xi}^{\left(t_{l}\right)}(t) .
$$

A more tractable expression is obtained by taking the Laplace transform of this new expression with respect to $t_{a}$ to obtain

$$
\frac{d}{d t} \hat{\Phi}_{\xi}^{(s)}(t)=-e^{s t}\left[\hat{\psi}^{*}(s)-\int_{0}^{t} d y e^{-s y} \psi^{*}(y)\right]+\hat{\psi}^{*}(s) \frac{d}{d t} \hat{\Phi}_{\xi}^{(s)}(t),
$$

from which it follows that

$$
\frac{d}{d t} \hat{\Phi}_{\xi}^{(s)}(t)=-\frac{e^{s t}\left[\hat{\psi}^{*}(s)-\int_{0}^{t} d y e^{-s y} \psi^{*}(y)\right]}{1-\hat{\psi}^{*}(s)} .
$$

The right-hand side of this equation appears formidable, but one can easily see by Laplace transforming Eq. (44) with respect to $t_{a}$, that it is nothing more than the Laplace transform with respect to $t_{a}$ of $-\psi_{t_{a}}^{*}(t)$, namely $-\hat{\psi}_{s}^{*}(t)$. Consequently, by inverse Laplace transforming Eq. (48) we obtain

$$
\frac{d}{d t} \Phi_{\xi}^{\left(t_{a}\right)}(t)=-\psi_{t_{a}}^{*}(t)
$$

which, by integration, yields

$$
\Phi_{\xi}^{\left(t_{a}\right)}(t)=\Phi_{\xi}^{\left(t_{a}\right)}(0)-\int_{0}^{t} d t_{1} \psi_{t_{a}}^{*}\left(t_{1}\right)
$$

The initial value of the $t_{a}$-old correlation function can be determined by explicitly Laplace transforming Eq. (43), calculated at $t=0$, with respect to $t_{a}$, thereby yielding

$$
\hat{\Phi}_{\xi}^{(s)}(0)=\hat{\Psi}^{*}(s)+\hat{\psi}^{*}(s) \hat{\Phi}_{\xi}^{(s)}(0),
$$

which simplifies to

$$
\left[1-\hat{\psi}^{*}(s)\right] \hat{\Phi}_{\xi}^{(s)}(0)=\hat{\Psi}^{*}(s) .
$$

On the other hand, we know that $\hat{\Psi}^{*}(s)=\left[1-\hat{\psi}^{*}(s)\right] / s$, so the initial Laplace variable is 


$$
\hat{\Phi}_{\xi}^{(s)}(0)=1 / s .
$$

It is obvious that the inverse Laplace transform of this last expression yields the initial condition for the correlation function $\hat{\Phi}_{\xi}^{\left(t_{a}\right)}(0)=1$, as it must be, based on the definition of Eq. (1) when $t_{1}=t_{2}$ and $\xi= \pm 1$, a dichotomous variable. Using this initial condition in (50), it follows that

$$
\Phi_{\xi}^{\left(t_{a}\right)}(t)=1-\int_{0}^{t} d t_{1} \psi_{t_{a}}^{*}\left(t_{1}\right)=\Psi_{t_{a}}^{*}(t)
$$

which, as we know from Sec. II, coincides with the exact expression for the $t_{a}$-old correlation function of Ref. [14].

\section{Approximation through iterative expansion, and truncation}

Although we established that Eq. (43) is exact, due to its implicit nature, it cannot be used to obtain an analytic function without carrying out a reasonable approximation. The approximation we select rests on replacing $\Phi_{\xi}^{\left(t_{t}\right)}(t)$ in the right-hand side of Eq. (43) with the function $A^{\left(t_{a}\right)}(t)$, which denotes a correlation function of uncertain age, which is, however, younger than the $t_{a}$-old correlation function defined by Eq. (43). This correlation function reads

$$
A^{\left(t_{a}\right)}(t)=\frac{\Phi_{\xi}(t)-\Phi_{\xi}\left(t+t_{a}\right)}{1-\Phi_{\xi}\left(t_{a}\right)} .
$$

The easiest way to derive (53) is to adopt the language of conditional probabilities. The numerator of this expression is the probability of not finding an event between 0 and $t$, minus the probability of not finding an event between $-t_{a}$ and $t$. If we call $A$ the condition of no event in the interval $(0, t)$, and $B$ the condition of at least one event in the interval $\left(-t_{a}, 0\right]$, then the numerator of the right-hand side of (53) can be identified with the joint probability $P(A, B)$. On the other hand, the denominator of (53) is simply $P(B)$, the probability of the event $B$ occuring, and therefore the function $A^{\left(t_{a}\right)}(t)$ can be identified, as it should, with the conditional probabilily $P(A \mid B)$, namely with the probability of finding no event between 0 and $t$ (i.e., it is a correlation function), given an event between $-t_{a}$ and 0 (i.e., it has an age younger than $t_{a}$ ).

Finally, by plugging Eq. (53) into Eq. (43) we obtain

$$
\Phi_{\xi}^{\left(t_{a}\right)}(t)=\Psi^{*}\left(t+t_{a}\right)+\left[1-\Psi^{*}\left(t_{a}\right)\right] \frac{\Phi_{\xi}(t)-\Phi_{\xi}\left(t+t_{a}\right)}{1-\Phi_{\xi}\left(t_{a}\right)} .
$$

This expression is not exact, but it is analytic. Moreover, the form of Eq. (43) suggests an iterative approach, and we can therefore refine our result, by replacing $\Phi_{\xi}^{\left(t_{1}\right)}(t)$ with $A^{\left(t_{a}\right)}(t)$, after an arbitrary number of iterations. In Fig. 1 we check the accuracy of the first-order approximation to the exact expression given by (54), by comparing it to the exact prediction of Eq. (40). The curves in Fig. 1 represent a numerical treatment of (40) with different values of $t_{a}$. In this example $\mu$ $=2.5$ and $T=1.5$ (as said, $\lambda=1$ ). The highest curve in the figure represents the stationary correlation function, namely

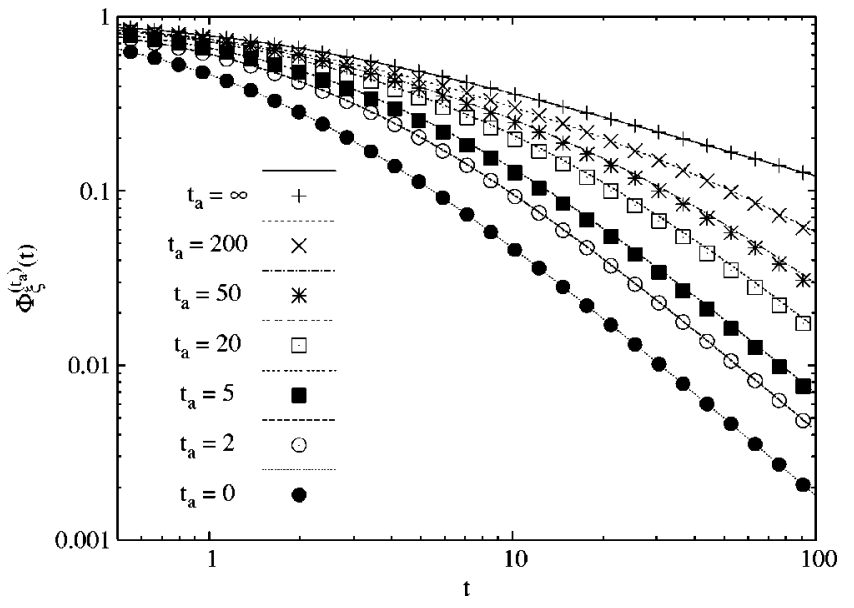

FIG. 1. The $t_{a}$-old correlation function, $\Phi_{\xi}^{t_{a}}(t)$, for different values of $t_{a}$. Curves represent a numerical integration of (40) with $z$ $=5 / 3$ and $T=1.5$, while dots correspond to the approximate formula (54).

$t_{a}=\infty$. The correlation function $\Phi_{\xi}^{\left(t_{a}\right)}(t)$ has a faster decay, with decreasing values of $t_{a}$. Finally, the lowest curve represents the correlation function with zero age, namely $\Psi_{t_{a}}^{*}(t)$. The symbols in Fig. 1 overlaying the continuous curves represent the calculations using Eq. (54) with various ages. We see perfect agreement for $t_{a} \leqslant\langle t\rangle$ and for $t_{a} \gg\langle t\rangle$. The agreement remains good for intermediate values, with discrepancies comparable to the numerical round-off errors.

\section{PHYSICAL CONSEQUENCES OF THE RESULTS OBTAINED}

The results of the earlier sections establish that the GME of arbitrary age, proposed in this paper, Eq. (30), fits the requirement of complete equivalence with the current literature on non-Poisson renewal processes. It is important to stress that a significant model, generating non-Poisson statistics from a renewal process, has been proposed by Bouchaud and others $[25,26]$. This model has raised wide interest and has recently been applied to a variety of pheonomena [27-31].

The Bouchaud trap model refers to the spin-glass dynamics, which is modeled with a set of branches, with different energies $E$, having a distribution

$$
p(E)=\beta_{g} \exp \left(-\beta_{g} E\right),
$$

where $T_{g} \equiv 1 / \beta_{g}$ is the glass transition temperature. For each branch this model assumes the Arrhenius prescription

$$
t=t_{0} \exp (\beta E),
$$

where $T \equiv 1 / \beta$ denotes the sample temperature, and $t$ the time necessary to overcome the barrier of intensity $E$, by means of thermal fluctuations.

Thus, using the relationship between the energy distribution and the waiting-time distribution, 


$$
\psi(t) d t=p(E) d E,
$$

after some simple algebra, these authors [25,26] obtain an inverse power-law waiting time distribution

$$
\psi(t)=\nu \frac{t_{0}^{\nu}}{t^{1+\nu}}
$$

where the power-law index is given by

$$
\nu \equiv \frac{\beta_{g}}{\beta} .
$$

For $\beta>\beta_{g}$ the resulting waiting-time distribution asymptotically corresponds to that of Eq. (32), with $\mu<2$. In this case, the stationary GME, namely, the infinitely aged master equation, does not exist, and we have to consider conditions for the system always being out of equilibrium. The approach discussed herein does not have any limitation, even if it is inspired to original work done moving from the stationary condition [see Eq. (7)].

It is important to stress that there exists increasing interest in understanding the blinking quantum dot phenomena. In addition to the paper by Jung et al. [10], we mention here more recent references [9,11,32-37]. The work of Brokman et al. [9] proves that these processes undergo the same form of aging as the Bouchaud trap model, thereby involving, also in this case, the non-Poisson renewal perspective. It is important to stress that in this field the recent work of Veberk et al. [37], addressing explicitly the issue of the intermittent fluorescence of blinking quantum dots, rests on a model that turns out to be equivalent to the Bouchaud trap model. In fact, according to [37], the electron responsible for the fluorescent emission is trapped at a distance $r$ with probability

$$
p(r)=a \exp \left(-\alpha_{p} r\right) \text {. }
$$

The recovery probability is

$$
k(r)=\left(1 / t_{0}\right) \exp \left(-\alpha_{k} r\right),
$$

implying that the electron is trapped for a time $t$ given by

$$
t=t_{0} \exp \left(\alpha_{k} r\right) \text {. }
$$

It is evident that this model is equivalent to the Bouchaud trap model. To realize this fact it is enough to replace the energies $E$ of the Bouchaud model with the distances $r$ of the model of Ref. [37]. The conclusion is that also in this case an inverse power law is generated,

$$
\psi(t)=\nu \frac{t_{0}^{\nu}}{t^{1+\nu}}
$$

where

$$
\nu=\frac{\alpha_{p}}{\alpha_{k}} .
$$

If $\alpha_{p}<\alpha_{k}$, we obtain a waiting time distribution with a diverging first moment, corresponding to the blinking quantum dot condition [37].

To move from the physics of spin glasses to the physics of quantum dots, as we plan to do in future publications with the help of the results of this paper, it becomes important to address fundamental issues such as the emission and absorption process. This leads us naturally to using the GME we derived in an previous section. If an out of equilibrium condition is created at a given time $t_{a}$, then after the preparation of the material, we predict the relaxation process of the quantity

$$
\Pi(t)=p_{1}(t)-p_{2}(t)
$$

to be driven by

$$
\frac{d}{d t} \Pi(t)=-\int_{0}^{t} d t^{\prime} \Phi_{t_{a}}\left(t-t^{\prime}\right) \Pi\left(t^{\prime}\right) d t^{\prime} .
$$

It is important to stress that this result rests on a GME that has an origin different from the prime principle procedure adopted, for instance, by Zwanzig [12], being essentially based on experimental observation. Is this compatible or not with a Liouville or Liouville-like treatment? This is an important issue that is discussed in the next section.

\section{CONCLUDING REMARKS}

The central result of this paper is the generalization of the GME discussed in Ref. [7] to one of arbitrary age. Another result, closely related to the GME of arbitrary age, is the generalization of the Onsager principle to physical conditions of any age. The validity of this generalization of the Onsager principle is confirmed by the fact that Eq. (19), the generalized Onsager principle, yields Eq. (18), and this is shown to be equivalent to the exact prescription of Godrèche and Luck, which is independently rederived in Sec. II C. Another interesting result is given by Eq. (54). This is an analytical expression for $t_{a}$-old correlation function, whose accuracy has been established using Eq. (40) which is another exact expression for the $t_{a}$-old correlation function, determined by the Liouville-like approach of Sec. III. In the special case of non-Poisson processes where the function $\psi^{*}(t)$ has the form given by Eq. (32), the approximate expression Eq. (54) turns out to be very accurate. Of course there might be non-Poisson processes where Eq. (54) is not as accurate as in the case presented here. However, the iterative procedure discussed in Sec. IV C, allows us to determine higher-order corrections, should they be necessary for a more satisfactory treatment.

It is important to understand why Eq. (54) is not exact, in spite of the fact that the approximation made for its derivation seems to fit the renewal nature of the process under study, where any jump resets memory to zero. This is a consequence of the infinite memory generated by non-Poisson dynamics, in spite of the fact the random events reset the memory to zero. The evaluation of the correlation function involves probabilistic arguments, and with them the infinite memory associated with the probabilistic treatment of nonPoisson processes.

We illuminate the meaning of the correlation function of arbitrary age by means of the Liouville-like density approach. The observation of the process of regression to equilibrium of the population difference corresponds to evaluating the antisymmetric distribution, while leaving the 
symmetric part of the distribution free to evolve. If the distribution is at equilibrium, the symmetric part corresponds to the equilibrium distribution and the integral of the left portion of the antisymmetric part, without back injection, regresses to zero as the corresponding equilibrium correlation function. For any other condition, the integral of the left portion of the antisymmetric part regresses to zero with an analytical expression depending on the time at which observation begins. The regression continues as a function of that specific initial condition while the symmetric part keeps moving towards equilibrium independently of the population difference. This explains why the regression to equilibrium depends on the initial condition, of any age, with no further dependence on the bath dynamics that keeps drifting towards equilibrium. This also explains why an emission or absorption spectrum [11] is not stationary and changes with time. The resonant radiation establishes a connection between the antisymmetric and the symmetric parts of the distribution, thereby updating observation to the changing bath conditions.

It is worth ending this paper with some further remarks about these theoretical problems. We have built up a GME of arbitrary age, using an empirical approach. Is it possible to derive the same GME by using a Liouville-like approach? In principle, we should use the Liouville-like picture of Sec. III, to derive, via contraction on the bath variables, the same GME, of arbitrary age, as that of Sec. II D. However, it is evident that this effort, even if we were successful, would be of limited help, for practical purposes. Suppose, for instance, that we have to study the response of the system to an external, time dependent, perturbation. Would the GME of arbitrary, but fixed, age, be useful for this purpose? It is evident that it would not. In fact, the external perturbation at times different from the age of the system would produce effects departing from the more realistic approach resting on perturbing trajectories. In the specific case of the absorption spectrum of blinking quantum dots [11] the authors, in fact, adopted this trajectory perspective to make a theoretical prediction that is incompatible with the perturbation of a GME of fixed age.
There already exists in the literature at least the discussion of one case [19] that seems to be a natural consequence of this property. Sokolov, Blumen, and Klafter [19] derived an exact density equation to describe a subdiffusion process. This equation corresponds to brand new initial conditions. This means a condition where $t_{a}=0$. Then, these authors perturbed this equation with a time-dependent field, and found that the theoretical result conflicts with the behavior of the CTRW under the influence of the same perturbation. This apparent contradiction arises because the time dependent perturbation corresponds to additional observations, taking place at different time values, none of them coinciding with the observation time, but the perturbation at $t=0$. This is true, whatever the observation time is, either $t_{a}=0$, as in Ref. [19], or $\infty>t_{a}>0$, a condition requiring the GME of this paper. Regardless of the observation time that we assign to the GME, it is impossible to make the GME prediction identical to the CTRW prediction, if we require the perturbation to remain external to the system. The concept of perturbation itself turns out to be inadequate to study non-Poisson processes, regardless of its intensity. Thus the results of the present paper, in addition to shedding light onto the implications of Ref. [19], imply a violation of linear response theory. The only possible way to make the density compatible with the trajectory picture is to make the external perturbation become a part of the system under study. This means that we have to build up a totally new, field-dependent, GME, along the lines of Ref. [11]. This sets a limit on the applicability of the GME of arbitrary age found in this paper. However, this result seems to support the conclusion that the trajectorydensity conflict, revealed by Bologna, Grigolini and West [13] might be a consequence of the aging properties emerging from non-Poisson renewal process.

\section{ACKNOWLEDGMENTS}

G.A. and P.G. thankfully acknowledge ARO for financial support through Grant DAAD19-02-1-0037. P.G. acknowledges Welch for financial support through Grant 70525.
[1] L. C. E. Struick, Physical Aging in Amorphous Polymers and Other Materials (Elsevier, Houston, 1978).

[2] L. Onsager, Phys. Rev. 38, 2265 (1931); 37, 405 (1931).

[3] C. Monthus and J.-P. Bouchaud, J. Phys. A 29, 3847 (1996).

[4] L. Laloux and P. Le Doussal, Phys. Rev. E 57, 6296 (1998).

[5] A. Mauger and N. Pottier, Phys. Rev. E 65, 056107 (2002).

[6] E. Barkai, Phys. Rev. Lett. 90, 104101 (2003).

[7] P. Allegrini, G. Aquino, P. Grigolini, L. Palatella, and A. Rosa, Phys. Rev. E 68, 056123 (2003).

[8] E. W. Montroll and B. J. West, in Fluctuation Phenomena edited by E. W. Montroll and J. L. Lebowitz (North-Holland, Amsterdam, 1979), pp. 61-177.

[9] X. Brokmann, J.-P. Hermier, G. Messin, P. Desbiolles, J.-P. Bouchaud, and M. Dahan, Phys. Rev. Lett. 90, 120601 (2003).

[10] Y.-J. Jung, E. Barkai, and R. Silbey, Chem. Phys. 284, 181 (2002).
[11] G. Aquino, L. Palatella, and P. Grigolini, Phys. Rev. Lett. 93, 050601 (2004).

[12] R. Zwanzig, in Lectures in Theoretical Physics Vol. 3, Boulder, Colorado, 1960, edited by W. E. Brittin, B. W. Downs, and J. Downs (Interscience, New York, 1961), p. 106.

[13] M. Bologna, P. Grigolini, and B. J. West, Chem. Phys. 284, 115 (2002).

[14] C. Godrèche and J. M. Luck, J. Stat. Phys. 104, 489 (2001).

[15] G. Margolin and E. Barkai, J. Chem. Phys. 121, 1566 (2004).

[16] V. M. Kenkre, E. W. Montroll, and M. F. Shlesinger, J. Stat. Phys. 9, 45 (1973).

[17] J. B. Bassingthwaighte, L. S. Liebovitch, and B. J. West, Fractal Physiology (Oxford University Press, Oxford, 1994).

[18] P. Allegrini, P. Grigolini, L. Palatella, and B. J. West, Phys. Rev. E 70, 046118 (2004).

[19] I. M. Sokolov, A. Blumen, and J. Klafter, Europhys. Lett. 56, 
175 (2001).

[20] P. Allegrini, P. Grigolini, L. Palatella, A. Rosa, and B. J. West, Physica A 347, 268 (2005).

[21] E. W. Montroll and G. W. Weiss, J. Math. Phys. 6, 167 (1965).

[22] G. Zumofen and J. Klafter, Phys. Rev. E 47, 851 (1993).

[23] G. Aquino, M. Bologna, P. Grigolini, and B. J. West, Phys. Rev. E 70, 036105 (2004).

[24] M. Ignaccolo, P. Grigolini, and A. Rosa, Phys. Rev. E 64, 026210 (2001).

[25] J. P. Bouchaud, J. Phys. I 2, 1705 (1992).

[26] J. P. Bouchaud and D. S. Dean, J. Phys. I 5, 265 (1995).

[27] M. Rokni and P. Chandra, Phys. Rev. B 69, 094403 (2004).

[28] C. Monthus, Phys. Rev. E 68, 036114 (2003).

[29] S. M. Fielding and P. Sollich, Phys. Rev. E 67, 011101 (2003).

[30] G. Ben Arous, A. Bovier, and V. Gayrard, Phys. Rev. Lett. 88,
087201 (2002).

[31] L. R. Fontes, M. Isopi, C. M. Newman, and D. L. Stein, Phys. Rev. Lett. 87, 110201 (2001).

[32] E. Barkai, Y.-J. Jung, and R. Silbey, Annu. Rev. Phys. Chem. 55, 457 (2004).

[33] I. Chung and M. G. Bawendi, Phys. Rev. B 70, 165304 (2004).

[34] J. Muller, J. M. Lupton, A. L. Rogach, J. Feldmann, D. V. Talapin, and H. Weller, Phys. Rev. Lett. 93, 167402 (2004).

[35] M. Pelton, D. G. Grier, and P. Guyot-Sionnest, Appl. Phys. Lett. 85, 819 (2004).

[36] F. Cichos, J. Martin, and C. von Borczyskowski, Phys. Rev. B 70, 115314 (2004).

[37] R. Verberk, Antoine M. van Oijen and Michel Orrit, Phys. Rev. B 66, 233202 (2002). 